Nonl. Analysis and Differential Equations, Vol. 2, 2014, no. 2, 83 - 90

HIKARI Ltd, www.m-hikari.com http://dx.doi.org/10.12988/nade.2014.421

\title{
An Abstract Approach to Nonlinear Boundary Value Problems at Resonance
}

\author{
Dan Maroncelli \\ Department of Mathematics \\ Concordia University St. Paul \\ 1282 Concordia Avenue \\ St. Paul, MN 55104-5494, USA
}

Copyright (c) 2014 Dan Maroncelli. This is an open access article distributed under the Creative Commons Attribution License, which permits unrestricted use, distribution, and reproduction in any medium, provided the original work is properly cited.

\begin{abstract}
In this paper we provide an abstract existence result for nonlinear boundary value problems at resonance. Our abstract approach provides a general framework for the situation that occurs for a large class of differential operators. We will approach the problem using an Alternative Method in conjunction with topological degree theory.
\end{abstract}

Mathematics Subject Classification: 34B15

Keywords: Boundary Value Problems, Resonance, Lyapunav-Schmidt, Topological degree theory

\section{Introduction}

In, [1], Cesari and Kannan prove an abstract existence theorem for a class of "selfadjoint" differential operators. Throughout they assume the nonlinearity involved is bounded. In this paper we extend the abstract existence result proved in [1] to a more general "non-selfadjoint" case in which the nonlinearity is also allowed to be unbounded. 


\section{Preliminaries}

Let $S$ denote an arbitrary Hilbert space. We will use $<\cdot, \cdot>$ to denote the inner product on $S$ and $\|\cdot\|$ to denote the norm induced by this inner product. We will also use $|\cdot|$ to denote the standard Euclidean norm on $\mathbb{R}^{n}$.

Let $\mathcal{L}: \operatorname{dom}(\mathcal{L}) \subset S \rightarrow S$ be a linear map which satisfies $0<\operatorname{dim}(\operatorname{ker}(\mathcal{L}))=$ $\operatorname{dim}(\operatorname{coker}(\mathcal{L}))<\infty$, where $\operatorname{coker}(\mathcal{L})$ denotes $S / \operatorname{Im}(\mathcal{L})$. We let $P: S \rightarrow S$ denote the orthogonal projection of $S$ onto $\operatorname{ker}(\mathcal{L})$ and $E: S \rightarrow S$ the orthogonal projection of $S$ onto $\operatorname{Im}(\mathcal{L})$. It is trivial that $\mathcal{L}$ restricted to $\operatorname{Ker}(P) \cap \operatorname{dom}(\mathcal{L})$ is one-to-one and onto $\operatorname{Im}(\mathcal{L})$. We let $M_{p}$ donote the inverse of $\mathcal{L}_{\left.\right|_{\operatorname{Ker}(P) \cap \operatorname{dom}(\mathcal{L})}}$. We will assume that $M_{p}$ is a compact linear operator on $S$.

We would like to remark that the properties of $\mathcal{L}$ listed above are a natural abstraction of the properties which occur in practice for a large class of differential operators. A special case of particular interest is the class of selfadjoint linear operators which occur in the study of Sturm-Liouville boundary value problems. For those readers interested in study of nonlinear boundary value problems with concrete instances of the differential operators described above, we suggest $[4,5,6,7,8,9,11]$

Let $\mathcal{F}: S \rightarrow S$ be a nonlinear continuous operator. We will be concerned with the existence of solutions to the operator equation

$$
\mathcal{L} x=\mathcal{F}(x) .
$$

Our approach will be to study the operator equation, (1), using the following system of equations

$$
\left\{\begin{array}{c}
(I-P) x-M_{p} E \mathcal{F}(x)=0 \\
\text { and } \\
(I-E) \mathcal{F}(x)=0
\end{array} .\right.
$$

The construction of this equivalent system is often referred to as the LyapunovSchmidt procedure. Readers interested in the details of this procedure or more general Alternative Methods may consult [2, 3, 6, 10].

If we let $v_{1}, v_{2}, \cdots, v_{n}$ and $w_{1}, w_{2}, \cdots, w_{n}$ denote orthonormal bases for $\operatorname{ker}(\mathcal{L})$ and $\operatorname{Im}(\mathcal{L})^{\perp}$ respectively, then $(2)$ is equivalent to

$$
\left\{\begin{array}{c}
u-M_{p} E \mathcal{F}(c v+u)=0 \\
\text { and } \\
\langle\mathcal{F}(c v+u), w\rangle=0
\end{array}\right.
$$

where $c \in \mathbb{R}^{n}, u \in \operatorname{Im}(I-P), c v$ denotes $\sum_{i=1}^{n} c_{i} v_{i}$ and $\langle\mathcal{F}(c v+u), w\rangle$ is notation 
for

$$
\left[\begin{array}{c}
\left\langle\mathcal{F}(c v+u), w_{1}\right\rangle \\
\left\langle\mathcal{F}(c v+u), w_{2}\right\rangle \\
\vdots \\
\left\langle\mathcal{F}(c v+u), w_{n}\right\rangle
\end{array}\right]
$$

We define an operator $H: \operatorname{Im}(I-P) \times \mathbb{R}^{n} \rightarrow \operatorname{Im}(I-P) \times \mathbb{R}^{n}$ by

$$
H(u, c)=\left[\begin{array}{c}
M_{p} E \mathcal{F}(c v+u) \\
c-\langle\mathcal{F}(c v+u), w\rangle
\end{array}\right] .
$$

From our discussion above, solving (1) is equivalent to finding the fixed points of $H$.

\section{Main Results}

In, [1], Cesari and Kannan prove the existence of solutions to (1), in the "selfadjoint" case, under the following two assumptions:

1. There is a constant $J_{0}$ such that $\|\mathcal{F}(x)\| \leq J_{0}$ for each $x \in S$.

2. There is a constant $R_{0} \geq 0$ such that for all $x \in S$ with $\|P x\| \geq R_{0}$ we have $\langle\mathcal{F}(x), P x\rangle \geq 0$ (or $\langle\mathcal{F}(x), P x\rangle \leq 0)$, whenever $\|(I-P) x\| \leq$ $\left\|M_{p} E\right\| J_{0}$.

In what follows, we extend these ideas to the case when $\mathcal{L}$ is not necessarily "selfadjoint". We also allow $\mathcal{F}$ to be unbounded, but assume it must satisfy a sublinear growth condition.

We start with a lemma which will play a significant role in our proof of the main result.

Lemma 3.1. Suppose $\mathcal{F}$ is sublinear, i.e. $\|\mathcal{F}(x)\| \leq M_{1}\|x\|^{\beta}+M_{2}$ where $0 \leq \beta<1$ and $M_{1}>0$. We then have that there exists positive constants, $C_{1}$ and $C_{2}$, such that for each pair $(u, c) \in \operatorname{Im}(I-P) \times \mathbb{R}^{n}$ satisfying $u=$ $M_{p} E F(c v+u)$ and $|c| \geq C_{1}$,

$$
\|u\| \leq C_{2}\|c v\|^{\beta} .
$$


Proof.

$$
\begin{aligned}
\|u\| & \leq\left\|M_{p} E\right\|\left(M_{1}(\|c v\|+\|u\|)^{\beta}+M_{2}\right) \\
& =\left\|M_{p} E\right\| M_{1}\|c v\|^{\beta}\left(1+\frac{\|u\|}{\|c v\|}\right)^{\beta}+\left\|M_{p} E\right\| M_{2} \\
& \leq\left\|M_{p} E\right\| M_{1}\|c v\|^{\beta}\left(1+\beta \frac{\|u\|}{\|c v\|}\right)+\left\|M_{p} E\right\| M_{2} .
\end{aligned}
$$

Thus,

$$
\frac{\|u\|}{\|c v\|^{\beta}}-\left\|M_{p} E\right\| M_{1} \beta \frac{\|u\|}{\|c v\|} \leq\left\|M_{p} E\right\| M_{1}+\frac{\left\|M_{p} E\right\| M_{2}}{\|c v\|^{\beta}}
$$

or

$$
\frac{\|u\|}{\|c v\|^{\beta}}\left(1-\frac{\left\|M_{p} E\right\| M_{1} \beta}{\|c v\|^{1-\beta}}\right) \leq\left\|M_{p} E\right\| M_{1}+\frac{\left\|M_{p} E\right\| M_{2}}{\|c v\|^{\beta}} .
$$

Now fix $\gamma$, with $0<\gamma<1$. If $|c| \geq\left(\frac{\left\|M_{p} E\right\| M_{1} \beta}{\gamma}\right)^{\frac{1}{1-\beta}}$, then

$$
\frac{\|u\|}{\|c v\|^{\beta}} \leq \frac{\left\|M_{p} E\right\| M_{1}}{1-\gamma}+\frac{\left\|M_{p} E\right\| M_{2}}{(1-\gamma)\left|\frac{\left\|M_{p} E\right\| M_{1} \beta}{\gamma}\right|^{\frac{\beta}{1-\beta}}}
$$

The result now follows by taking

$$
C_{1}=\left(\frac{\left\|M_{p} E\right\| M_{1} \beta}{\gamma}\right)^{\frac{1}{1-\beta}} \text { and } C_{2}=\frac{\left\|M_{p} E\right\| M_{1}}{1-\gamma}+\frac{\left\|M_{p} E\right\| M_{2}}{(1-\gamma)\left|\frac{\left\|M_{p} E\right\| M_{1} \beta}{\gamma}\right|^{\frac{\beta}{1-\beta}}}
$$

Remark 3.2. We will not be concerned with the case of bounded $\mathcal{F}$, this is the main reason for assuming $M_{1}>0$ in Lemma 3.1. We would, however, like to point out that in the case of bounded $\mathcal{F}$, we get the more desirable result:

If $(u, c) \in \operatorname{Im}(I-P) \times \mathbb{R}^{n}$ satisfying $u=M_{p} E \mathcal{F}(c v+u)$, then there exists a $J_{0}$ such that

$$
\|u\| \leq\left\|M_{p} E\right\| J_{0} .
$$

The proof of the existence theorem found in [1] relies indispensably on this observation. 
We now proceed to the proof of the main result. The following observation will be used in what follows: there exists $r^{*}$ such that for all $r \geq r^{*}$, we have

$$
r-\left\|M_{p} E\right\|\left(M_{1} 2^{\beta} r^{\beta}+M_{2}\right)>0 .
$$

We will assume that $r^{*}$ has been chosen such that $C_{1}$ (Lemma 3.1) satisfies $r^{*}>C_{1}$.

Theorem 3.3. Suppose the following conditions hold:

C1. $\mathcal{F}$ is "sublinear"; that is, there exist real numbers $M_{1}, M_{2}$ and $\beta$, with $0 \leq \beta<1$ and $M_{1}>0$, such that $\|\mathcal{F}(x)\| \leq M_{1}|x|^{\beta}+M_{2}$.

C2. There is a constant $R_{0} \geq r^{*}$ such that for all $x=c v+u \in S$ with $|c| \geq R_{0}$ we have $\langle\mathcal{F}(x), c w\rangle \geq 0$ (or $\langle\mathcal{F}(x), c w\rangle \leq 0)$, whenever $\|u\| \leq C_{2} R_{0}^{\beta}$. (Here $C_{2}$ is as in Lemma 3.1).

Then the operator equation, (1), has at least one solution.

Proof. We will assume at first that $\langle\mathcal{F}(x), c w\rangle \geq 0$. In order to apply a degree theoretic argument, we make $\operatorname{Im}(I-P) \times \mathbb{R}^{n}$ a Banach space using the following norm

$$
\|(u, c)\|=\max \{\|u\|,|c|\} .
$$

We then define an operator $H: \operatorname{Im}(I-P) \times \mathbb{R}^{n} \rightarrow \operatorname{Im}(I-P) \times \mathbb{R}^{n}$ by

$$
H(u, c)=\left[\begin{array}{c}
M_{p} E \mathcal{F}(c v+u) \\
c-\langle\mathcal{F}(c v+u), w\rangle
\end{array}\right] .
$$

We know, from our earlier discussion, that the fixed points of $H$ are precisely the solutions of the operator problem (1). Since $M_{p}$ is compact and $\mathcal{F}$ is continuous and sublinear, we have that $H$ is a nonlinear compact map. We will show that $H$ has a fixed point by showing that for an appropriately chosen set, the Leray-Schauder degree of $I-H$ is nonzero.

We define

$$
\Omega=B\left(0, R_{0}\right) \text { in } \operatorname{Im}(I-P) \times \mathbb{R}^{n},
$$

and

$Q:[0,1] \times \bar{\Omega} \rightarrow \operatorname{Im}(I-P) \times \mathbb{R}^{n}$ 
by

$$
Q(\lambda,(u, c))=\left[\begin{array}{c}
u-\lambda M_{p} E F(c v+u) \\
(1-\lambda) c+\lambda\langle\mathcal{F}(c v+u), w\rangle
\end{array}\right] .
$$

It is clear that $Q$ is a homotopy between $I$ and $I-H$. We will analyze $Q$ on the boundary of $\Omega$ which we denote by $\partial(\Omega)$. true:

It is clear that if $(u, c) \in \partial(\Omega)$, then one of two following condtitions is

i. $\|x\|=R_{0}$ and $|c| \leq R_{0}$.

ii. $\|x\| \leq R_{0}$ and $|c|=R_{0}$.

We first look at the case where $(x, \alpha) \in \partial(\Omega)$ and i. holds.

Since $R_{0}>r^{*}$, we have

$$
R_{0}-\left\|M_{p} E\right\|\left(M_{1} 2^{\beta} R_{0}^{\beta}+M_{2}\right)>0 .
$$

Now,

$$
\begin{aligned}
\|c v+u\| & \leq\|c v\|+\|u\| \\
& =|c|+\|u\| \\
& \leq 2 R_{0}
\end{aligned}
$$

so that

$$
\begin{aligned}
\left\|M_{p} E \mathcal{F}(c v+u)\right\| & \leq\left\|M_{p} E\right\|\left(M_{1}\left(2 R_{0}\right)^{\beta}+M_{2}\right) \\
& <R_{0}=\|u\| .
\end{aligned}
$$

We now assume $(u, c) \in \partial(\Omega)$ and that ii. holds. We may assume, without loss of generality, that $u=\beta M_{p} E \mathcal{F}(c v+u)$, for some $\beta \in(0,1)$.

Now,

$$
\begin{aligned}
& |(1-\lambda) c+\lambda\langle\mathcal{F}(c v+u), w\rangle|^{2}= \\
& \quad(1-\lambda)^{2}|c|^{2}+\lambda^{2}|\langle\mathcal{F}(c v+u), w\rangle|^{2}+2(1-\lambda) \lambda\langle c,\langle\mathcal{F}(c v+u), w\rangle\rangle_{\mathbb{R}},
\end{aligned}
$$

where $\left\langle\cdot, \cdot>_{\mathbb{R}}\right.$, denotes the standard Euclidean inner product. 
However,

$$
\begin{aligned}
\langle c,\langle\mathcal{F}(c v+u), w\rangle\rangle_{\mathbb{R}} & =\sum_{i=1}^{n} c_{i}\left\langle\mathcal{F}(c v+u), w_{i}\right\rangle \\
& =\left\langle\mathcal{F}(c v+u), \sum_{i=1}^{n} c_{i} w_{i}\right\rangle \\
& =\langle\mathcal{F}(c v+u), c w\rangle .
\end{aligned}
$$

Since

$$
\|c v\|=|c|=R_{0} \geq r^{*}>C_{1} \text { and } u=\beta M_{p} E \mathcal{F}(c v+u),
$$

we have

$$
\|u\| \leq C_{2}\|c v\|^{\beta} \leq C_{2} R_{0}^{\beta} .
$$

It follows that $\langle\mathcal{F}(c v+u), c w\rangle \geq 0$ and thus

$$
|(1-\lambda) c+\lambda\langle\mathcal{F}(c v+u), w\rangle|^{2}>0 .
$$

The proof is now complete, from homotopy invariance of the Leray-Schauder degree, by noticing that (4) and (5) show that $Q(\lambda,(u, c))$ is nonzero for each $(u, c) \in \partial(\Omega)$ and every $\lambda \in(0,1)$.

The case where $\langle\mathcal{F}(c v+u), c w\rangle \leq 0$ is handled in a similar manner by constructing a homotopy between $-I$ and $I-H$.

Remark 3.4. If we assume $\mathcal{L}$ is "self-adjoint", then it is clear that Theorem 3.3 is a natural generalization, to the case of an unbounded nonlinearity $\mathcal{F}$, of the result found in [1].

\section{References}

[1] L. Cesari and R. Kannan. An abstract existence theorem at resonance. Proc. Amer. Math. Soc, 63:221-225, 1977.

[2] S. Chow and J. K. Hale. Methods of Bifurcation Theory. Springer, Berlin, 1982.

[3] J. K. Hale. Ordinary Differential Equations. Robert E. Kreiger Publishing Company, Malabar FL, 1980.

[4] D. Maroncelli and J. Rodríguez. On the solvability of multipoint boundary value problems for discrete systems at resonance. Journal of Difference Equations and Applications, 10.1080/10236198.2013.805216, 2013. 
[5] D. Maroncelli and J. Rodríguez. Weakly nonlinear boundary value problems with impulses. Dyn. Contin. Discrete Impuls. Syst. Ser. A Math. Anal., accepted, 2013.

[6] D. Maroncelli and J. Rodríguez. On the solvability of nonlinear impulsive boundary value problems. Topol. Methods Nonlinear Anal., accepted.

[7] Jesús Rodríguez. Nonlinear discrete sturm-liouville problems. J. Math. Anal. Appl., 308, Issue 1:380-391, 2005.

[8] Jesús Rodríguez and Zarchary Abernathy. Nonlinear discrete sturmliouville problems with global boundary conditions. Differ. Eqn. Appl., 18, Issue 3:1-15, 2012.

[9] Jesús Rodríguez and Zarchary Abernathy. On the solvability of nonlinear sturm-liouville problems. J. Math. Anal. Appl., 387, Issue 1:310-319, 2012 .

[10] Jesús Rodríguez and Padraic Taylor. Multipoint boundary value problems for nonlinear ordinary differential equations. Nonlinear Anal., 68:34653474, 2008.

[11] Jesús F. Rodríguez. Existence theory for nonlinear eigenvalue problems. Appl. Anal., 87:293-301, 2008, no. 3.

Received: February 18, 2014 